\title{
A Formal Path Inference of Starch Biosynthesis via Mathematical Modelling of Metabolic Changes in Excess $\mathrm{CO}_{2}$
}

\author{
Treenut Saithong ${ }^{1,3 *}$, Asawin Meechai ${ }^{2,3}$, Supapon Cheevadhanarak ${ }^{1,3,4}$ and Sakarindr Bhumiratana ${ }^{2,3,5}$ \\ ${ }^{1}$ School of Bio resources and Technology, King Mongkut's University of Technology Thonburi, Thailand \\ ${ }^{2}$ Department of Chemical Engineering, King Mongkut's University of Technology Thonburi, Bangkok. Thailand \\ ${ }^{3}$ Systems Biology and Bioinformatics research group (SBI), King Mongkut's University of Technology Thonburi, Bangkuntien Campus, Bangkok, Thailand \\ ${ }^{4}$ Pilot Plant Development and Training Institute (PDTI), King Mongkut's University of Technology Thonburi, Bangkuntien Campus, Bangkok, Thailand \\ ${ }^{5}$ National Science and Technology Development Agency (NSTDA), Pathumthani, Thailand
}

\begin{abstract}
Great demand for plant starch has made starch biosynthesis one of the most studied pathways in the literature. Many attempts have been made to improve the yield and properties of starch, including research on $\mathrm{CO}_{2}$ elevation as a means of increasing production; however, the analyses often faced difficulty in transiently and simultaneously measuring the metabolites of interest. Our work aimed to break-through such restrictions by systematically investigating the changes in metabolism of starch throughout the pathway-from source to sink cells-with the aid of mathematical modelling. Monitoring changes in metabolite concentrations and flux distributions allowed us to propose a formal metabolic path (i.e. a preferential pathway in charge of a particular event) responsible for starchyield variation under excess carbon-substrate. Our findings not only supported many established hypotheses on the regulations of starch production, but also gave reasonable predictions of metabolic regulation of starch biosynthesis.
\end{abstract}

Keywords: Starch biosynthesis; Biological modelling; Formal path; Metabolic model; $\mathrm{CO}_{2}$ elevation

\section{Introduction}

Starch is a carbon storage metabolite of plants assembled from alpha 1, 4 linear glucan (amylose) and alpha 1, 6 branch glucan (amylopectin) [1,2]. Plant starch is not only the largest food resource, but is also a raw material for various applications, including biofuels [3], cosmetics [4], and medical mixture compounds [4]. Considering its diverse applications, quantity and quality of produced starch are equally important. While the amylose and amylopectin production process inside plant cells is partly known [5-8], the starch formation process from those components is not fully understood, as evident in the available metabolic network [1,9]. Such incomplete data prevent the understanding of the mechanism behind starch metabolism and impede starch-related strain improvement. Consequently, knowledge that would lead to enhancing the quality and quantity of plant starch is becoming increasingly crucial, given that starch is an essential food source for a growing world population.

Starch metabolism is a highly regulated process involving numerous enzymes and metabolites. The metabolic pathway of starch metabolism is complex and includes various types of cells that can be broken down into two classes: photosynthetic (source cells) and nonphotosynthetic (sink cells) cell types (Figure 1,2). In the presence of light, light trapping (light reaction of photosynthesis) and $\mathrm{CO}_{2}$ fixation (Calvin cycle: dark reaction of photosynthesis) occur in leaves, where the first process of carbon anabolism starts in plants. Through the Calvin cycle, a one-carbon molecule, $\mathrm{CO}_{2}$, is converted into a threecarbon molecule, triose phosphate including dihydroxy acetone phosphate (DHAP) and glyceraldehydes-3-phosphate (GAP). Triose phosphate can either be used as a substrate for the formation of larger molecules or directly utilised in regular cell activities. It has been shown that part of triose phosphate is used for transitory starch formation, which is accumulated in leaves during the day, while the remainder is directly converted into sucrose to serve in the glycolysis pathway or to transport to sink cells [10]. In darkness (non-photosynthetic pathway), transitory starch in leaves is broken down into sucrose via the starch degradation pathway, and the produced sucrose is subsequently mobilised through the phloem tube to storage organs (e.g., meristems, root cap cells, seeds, fruits, and tubers [7,11-15]), where the transported sucrose is converted into storage starch.

Many experiments to increase starch yield have experienced biological complexities such as the plasticity of metabolic modifications via enzyme-activity adjustment (e.g., modification of AGPase reviewed by (Smith, 2008)) and inconsistent measurements of data due to experimental limits [16-18]. At first, the efficiency of related enzymes was the main focus in an attempt to increase the starch yield, as seen in the extensive research on the ADP-glucose pyrophosphorylase enzyme (AGPase) $[14,16,19,20]$, which has been proposed as a starchproduction-limiting enzyme $[20,21]$. However, the recent research focus has moved toward substrate limitation [14,17,18,22-24] and other metabolic regulations [25].

One difficulty in understanding the regulation of starch metabolism lies in the high degree of collaboration between constituent pathways (e.g. photosynthesis, carbon assimilation throughout starch catabolism and anabolism) within/across cells (e.g. leaf cells, vascular cells, and storage cells), raising the issue of a highly systematic-controlled system. Experimental efforts needed to shed light on the systematic regulation would be enormous. Such biological complexity therefore challenges systems biologists to develop a mathematical model of the system that could facilitate experimental study.

${ }^{*}$ Corresponding author: Treenut Saithong, School of Bio resources and Technology, Systems Biology and Bioinformatics research group (SBI), King Mongkut's University of Technology Thonburi, 83 Moo 8 Thakam Bankhuntein Bangkok 10150, Thailand, Tel: +66 2470 7714; E-mail: treenut.sai@kmutt.ac.th

Received January 21, 2012; Accepted March 26, 2012; Published March 28, 2012

Citation: Saithong T, Meechai A, Cheevadhanarak S, Bhumiratana S (2012) A Formal Path Inference of Starch Biosynthesis via Mathematical Modelling of Metabolic Changes in Excess $\mathrm{CO}_{2}$. J Comput Sci Syst Biol 5: 024-037. doi:10.4172 jcsb.1000087

Copyright: (c) 2012 Saithong T, et al. This is an open-access article distributed under the terms of the Creative Commons Attribution License,which permits unrestricted use, distribution, and reproduction in any medium, provided the original author and source are credited. 
Citation: Saithong T, Meechai A, Cheevadhanarak S, Bhumiratana S (2012) A Formal Path Inference of Starch Biosynthesis via Mathematical Modelling of Metabolic Changes in Excess $\mathrm{CO}_{2}$. J Comput Sci Syst Biol 5: 024-037. doi:10.4172/jcsb.1000087

Mathematical modelling has been applied to unwind biological complexity for decades, and its success has been reported in various organisms, including plants [26-29]. The evolution of biological models begins with simple static models-which fail to accurately represent a natural living cell-but gradually leads to more complicated dynamic models containing more biological details. For plant metabolism, the scope of models is often limited by the boundary of the cell, such as photosynthesis models $[8,30,31]$ in leaf cells (or in chloroplast) and starch biosynthesis in tuber cells (or in amyloplast) $[6,7,32,33]$. In an extension of previous plant metabolic models, we incorporated the whole starch metabolism network into one model, aiming to monitor the global alterations of the metabolic pathway under perturbations. The compartmentalised modelling technique was therefore selected to construct a model of starch biosynthesis spanning across plant cells (leaf, phloem, and tuber cells). This model aimed at C3-plants, especially potatoes, which store starch in a tuber. The main information for the model involving the Calvin cycle and starch synthesis (in the storage organ) pathway were taken from $[5,8,17,31,34-36]$ and $[6,7,37,38]$, respectively. The validated model, according to the literature, was subsequently employed to investigate the effect of carbon-source elevation on metabolite levels in order to gain insight into the metabolic regulation controlling the yield of plant starch.

\section{Methods}

\section{Modelling strategy}

An architectural design of the model compartments is required to construct a multi-cellular model. In addition, the general pipeline of modelling consists of data collection, model development, model validation, and model prediction. Here, each cell type relevant to starch biosynthesis was designed as a separated compartment. The minimal model of starch metabolism was thus created by assembling three cell types together, including leaf cells, where the Calvin cycle takes place to fixate $\mathrm{CO}_{2}$ during photosynthesis; the phloem, where sucrose is transported to the tuber as a substrate for starch biosynthesis; and tuber cells, where permanent starch is formed and stored. Figure 1 illustrates the layout of the developed multi-cellular model of starch metabolism based on the aforementioned design.

\section{Model development}

The structured model of the three compartments (leaf cell, phloem, and tuber cell) covers five constituent pathways of starch metabolism (Figure 2): Calvin cycle (grey), transitory starch degradation (yellow), sucrose synthesis (green), sucrose transportation (black arrow), and permanent starch biosynthesis (blue). The biochemical reactions in each pathway were gathered from the literature. Sixteen reactions in the Calvin cycle were mainly collected from [8], while the other twenty-three reactions in the leaf cell-belonging to the transitory starch degradation and sucrose synthesis pathways-were gathered from many published data of carbon metabolism in the leaf $[5,35,36]$. The transport of sucrose in the phloem was simply modelled by a single reaction, and relied on Fick's first law of diffusion with a characteristic length of $4 \times 10^{-6}$ meters (estimated from the radius of the phloem sieve tube in cassava (Wongpayak, 2006)). For starch biosynthesis in the tuber, nineteen reactions were reconstructed by expanding our previously published model of the potato tuber [6] with the existing data $[7,37]$. In summary, the reconstructed starch metabolism network (Figure 2) contains 58 enzymatic reactions and 49 metabolites.

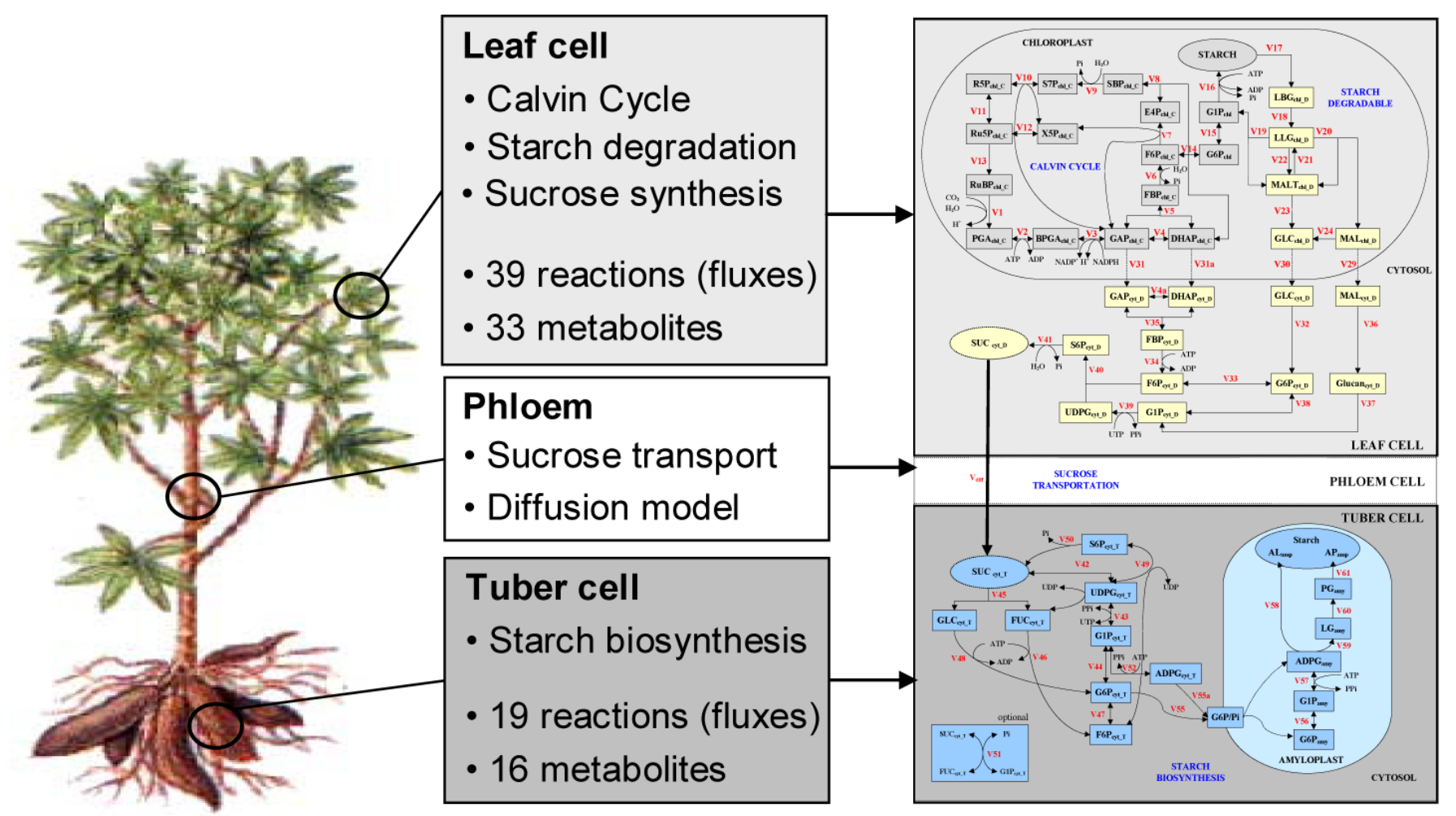

Figure 1: The architecture of the starch biosynthesis model. The scheme illustrates the architectural design of the compartmentalised model for starch biosynthesis comprised of 3 parts: leaf, phloem, and tuber. Each compartment contains constituent metabolic pathways in the starch production, which continuously lies across cell types (see also Figure 2). 
Citation: Saithong T, Meechai A, Cheevadhanarak S, Bhumiratana S (2012) A Formal Path Inference of Starch Biosynthesis via Mathematical Modelling of Metabolic Changes in Excess $\mathrm{CO}_{2}$. J Comput Sci Syst Biol 5: 024-037. doi:10.4172/jcsb.1000087

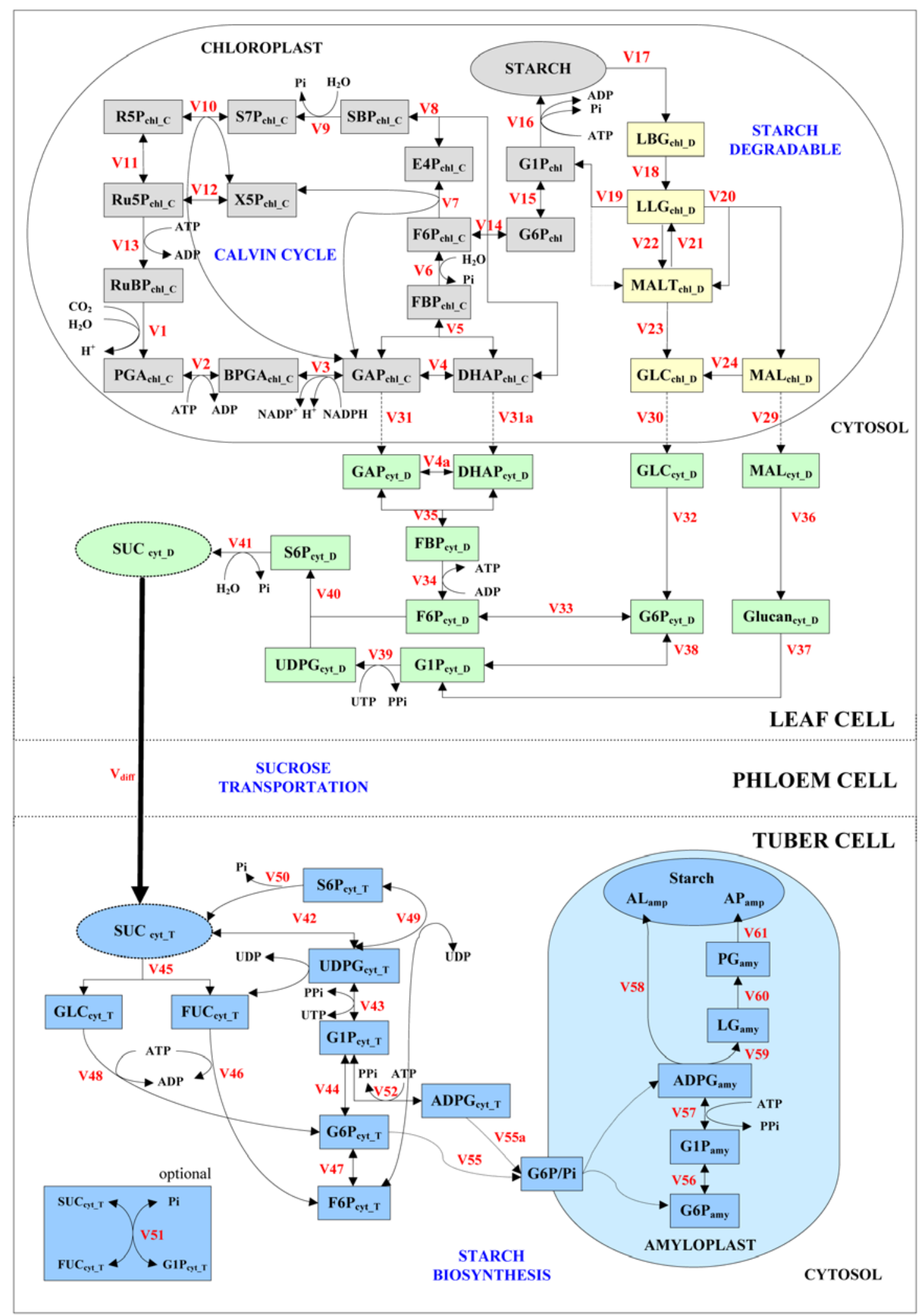

Figure 2: The overall pathway of starch biosynthesis. The scheme exhibits the scope of pathway under study, starting from $\mathrm{CO}_{2}$ fixation in the Calvin cycle and ending at amylose and amylopectin production. The constituent metabolic pathways in the starch production process are marked by colour tones: grey-Calvin cycle, yellow-starch degradation and sucrose synthesis, and blue-permanent starch synthesis. Metabolic reactions driven by enzymes (red texts) are represented by arrows $(\rightarrow)$, while metabolites are denoted by squares and ovals with black labels. The abbreviations of enzyme as well as metabolite names, and enzyme kinetic information used in the studied model are described in Appendix (Table A1) and supplementary data (Tables S1-S4), respectively. 
Despite an enormous effort focusing on cell imitation, the proposed models are always much less complex than the real mechanism inside the cell. A certain assumption is therefore required to define the scope of study, such as the boundary of the model, the condition of interest, and the model exception. These assumptions are crucial in defining the capability of the model and also in the interpretation of the simulated results. To that end, the developed model of starch metabolism was simulated under the following assumptions:

1. We modelled the metabolic pathways of starch metabolism from photosynthesis to tuber starch formation through the compartmentalised conceptual model, consisting of three types of cells: leaf cell (photosynthesis, transitory starch breakdown, and sucrose synthesis), phloem (sucrose transportation), and tuber cell (starch biosynthesis).

2. Light reaction in photosynthesis was excluded, so the model begins at carbon dioxide $\left(\mathrm{CO}_{2}\right)$ fixation in the Calvin cycle.

3. Light/dark cycle is an important factor affecting the starch biosynthesis process; however, the complexities in light perception of plants and the effect of light on the metabolism prevented us from taking the cycle into account in the current model. How plants perceive light and how light directs the metabolism are still open questions. Though a circadian clock is suggested to play the role in light/dark cycle measurement [39] and its model is available [40], there is no clear cascade of how the circadian clock is linked to the starch biosynthesis pathway. As a consequence, the kinetic parameter values were assumed to be constant throughout the simulation time, independent of time of the day (or the presence of light).

4. Due to an unclear mechanism in starch-granule assimilation, the model was terminated at the production of two main components of the starch granule: amylose and amylopectin.

5. The quantity of tuber starch (starch content) is suggested by the combined amount of the amylose and amylopectin, while the quality of the produced starch is implied by the ratio of these metabolites (AL/AP).

6. To simplify the elaborate metabolic network in real plants, only backbone reactions in the five pathways relevant to starch metabolism were modelled (see also Assumption 1), and an excess abundance of currency metabolites (e.g., ATP, ADP, Pi) was assumed.

7. The transportation process through the phloem-which links the source cell (photosynthetic cell) and the sink cell (non- photosynthetic cell) of the plant model-was described according to Fick's First law of Motion. The characteristic length (L) was approximately $4 \times 10^{-6}$ meter [41].

8. As with various metabolic models [27,28], Michaelis-Menten enzyme kinetics and its derivatives were mainly employed to describe behaviours of the constituent biochemical reactions.

Based on these assumptions, a set of ordinary differential equations [42] representing the metabolic network of starch metabolism can be written as exhibited in Tables S1-S2. Kinetic parameters required for simulating the model were collected or approximated from available published data and Brenda databases [43]. Some parameters-e.g. the typical ratio of starch composition (AL/AP; more detail is available in the supplementary data, Tables S3-S4)-were tuned to achieve a sensible simulation. The mathematical model was solved using Berkeley Madonna, a product of the University of California, Berkeley (http:// www.berkeleymadonna.com/ ).

\section{Model validation}

An important step following model development is model validation. This step aims at examining the suitability of the model by comparing simulated results with experimental data. The accuracy in the comparison indicates the ability of the model to represent the behaviours of real plants, and also suggests the degree of model predictability.

To validate the proposed kinetic model of starch metabolism, the model was perturbed at some investigated conditions at which the simulated results were qualitatively compared. Quantitative match will be carried out in the next version of the model in which the light/dark cycle is taken into account to fully imitate experimental conditions. All of the employed cases for model validation were selected from the available publications that relate to the systems of interest. Table 1 summarises the results of comparison between the model simulations and the corresponding measured data. The number of matches assures the goodness of the constructed model for further applications.

\section{Model simulation}

While the final state of metabolites (i.e., steady state fluxes or concentrations) under perturbation can be observed through molecular biological and biochemical techniques, the transient state of those (i.e., metabolic alteration) is hard to determine by such methods. For instance, a significant decrease in starch yield results from mutating the gene encoding the ADP-glucose pyrophosphorylase (ADPase) enzyme $[16,19]$. The activity of the perturbed enzyme (and/or the transcriptional level of the mutated gene) is always only a reported metabolic parameter,

\begin{tabular}{|l|l|l|}
\hline Events & Literature evidence & Simulation results \\
\hline $\begin{array}{l}\text { (1) Perturbation of aldolase enzyme } \\
\text { activity }\end{array}$ & $\begin{array}{l}\text { Haake et al. indicated that reduction in aldolase enzyme activity } \\
\text { affects various metabolite concentrations in the Calvin cycle, } \\
\text { leading to the increased concentrations of DHAP and GAP [53]. }\end{array}$ & $\begin{array}{l}\text { Decreased aldolase (V5) activity in the model results in } \\
\text { increased GAP and DHAP concentrations. }\end{array}$ \\
\hline $\begin{array}{l}\text { (2) Perturbation of transketolase } \\
\text { enzyme activity }\end{array}$ & $\begin{array}{l}\text { Henkes et al. presented that suppression of transketolase } \\
\text { enzyme activity causes a reduction in leaf sugars, since } \\
\text { photosynthesis is inhibited [54]. }\end{array}$ & $\begin{array}{l}\text { Decreased transketolase (V5) activity in the model results } \\
\text { in decreased photosynthetic sugars, GAP and DHAP } \\
\text { concentrations. }\end{array}$ \\
\hline $\begin{array}{l}\text { (3) Perturbation of DBE enzyme } \\
\text { activity }\end{array}$ & $\begin{array}{l}\text { Dinges et al. found that the deficiency in DBE encoding gene } \\
\text { results in accumulation of phytoglycogen (PG) or water soluble } \\
\text { polysaccharide (WSP) [55]. }\end{array}$ & $\begin{array}{l}\text { Decreased debranching enzyme activity (V61) in the model } \\
\text { results in increased PG concentrations. }\end{array}$ \\
\hline (4) $\mathrm{CO}_{2}$ elevation & $\begin{array}{l}\text { Lawson et al. showed that concentrations of several metabolites } \\
\text { in the Calvin cycle increase with the elevated CO }{ }_{2} \text { concentration, } \\
\text { for example PGA, DHAP, GAP [23]. }\end{array}$ & $\begin{array}{l}\text { Increased CO } \\
\text { increased concentrations of PGA, DHAP, and GAP. }\end{array}$ \\
\hline
\end{tabular}

Table 1: Summary of model validation. The table summarises the literature-based validation of the constructed model. The model simulations were compared with the published data measured under the corresponding conditions. 
basically measured to confirm the success of disruption that is expected to be the cause of the resulting phenotype. However, such an approach cannot describe how the altered enzyme activity affects the metabolism that subsequently leads to starch reduction. Without a clear description of what really happens at a metabolic level due to the mutation (or other disruptions), the given explanation is intuitively concluded from the expectation and experience of experimentalists. To alleviate the degree of arbitrariness and human biases, model simulation is thus exploited in providing a demonstration of the systematic alteration occurring in metabolism under perturbation, both during the transition state and at the steady state. Alterations of fluxes through enzymes and metabolite concentrations estimated from the model infer the response of the system to the applied perturbation, which is critical information for understanding the metabolic network.

Among the approaches to improve plant starch content for industrial demand $[3,12,16,22-24]$, doubling $\mathrm{CO}_{2}$ concentration in the plant culture is the logical first choice. As $\mathrm{CO}_{2}$ is a cheap and unlimited carbon source for plants, it is economically sensible to increase starch content in plants via elevation of $\mathrm{CO}_{2}$ uptake rate. Moreover, high $\mathrm{CO}_{2}$ usage of such plants allows them to be air cleaners (environmentally sensible) in addition to being starch producers. These reasons motivated us to investigate the metabolic process underlying the starchy phenotype of plants, cultured under high $\mathrm{CO}_{2}$ concentration conditions. By employing the developed kinetic model, dynamic changes inside the starch biosynthesis pathway in response to an increased $\mathrm{CO}_{2}$ concentration can be monitored through the simulation of fluxes and metabolite concentrations. In practice, the model that satisfies the literature-based validation (Table 1) was used to simulate the metabolic response under various $\mathrm{CO}_{2}$ concentrations $\left(4 \times 10^{-8}\right.$ to $\left.4 \times 10^{-4} \mathrm{mM}\right)$

Sets of metabolite concentrations and fluxes in the starch biosynthesis pathway were estimated by simulating the model under a series of $\mathrm{CO}_{2}$ concentrations, whereby the concentration of $\mathrm{CO}_{2}$ was fixed as a constant in the model for each simulation. Five different $\mathrm{CO}_{2}$ concentrations $\left(4 \times 10^{-8}, 4 \times 10^{-7}, 4 \times 10^{-6}, 4 \times 10^{-5}\right.$, and $\left.4 \times 10^{-4} \mathrm{mM}\right)$ produced five simulated datasets that were compared by a log ratio (Equation 1) with respect to the simulation under a minimal $\mathrm{CO}_{2}$ concentration $\left(=4 \times 10^{-8} \mathrm{mM}\right)$.

$$
\log \text { ratio }=\ln \left(\frac{\left.y_{i}\right|_{\mathrm{CO} 2 \neq 4 e-8}}{\left.y_{i}\right|_{\mathrm{CO} 2=4 e-8}}\right)
$$

In Equation (1), $y_{i}$ is the metabolic quantity (i.e. concentrations and fluxes) of the $i^{t h}$ component within total $n$ components in the pathway, simulated at certain $\mathrm{CO}_{2}$ concentrations $\left(4 \times 10^{-8}\right.$ to $\left.4 \times 10^{-4} \mathrm{mM}\right)$.

The simulated results used in the subsequent analysis were taken at $t=1000$ seconds, where almost all of the metabolic entities reached pseudo-steady sate, except the end-product metabolites, such as amylose and amylopectin. Figure 3 is a heat map plot of the log ratio inferring metabolic changes in response to $\mathrm{CO}_{2}$ elevation. The colour intensity represents the magnitude of metabolic alteration, whereas the colour tones denote the direction of change in metabolic quantities with an increased $\mathrm{CO}_{2}$ concentration: increase (from grey $(0)$ to red (maximum) ) and decrease (from grey (0) to green (maximum)). The result shows that increasing $\mathrm{CO}_{2}$ concentration significantly affects carbon metabolism in the leaf, especially in the Calvin cycle, whereas the effect is relatively low in the tuber (Figure 3,4). By focusing on metabolic adaptation at elevated $\mathrm{CO}_{2}$ concentrations, we observed biological information as presented in the next section.

\section{Results}

\section{Changes in metabolite concentrations and fluxes under elevated $\mathrm{CO}_{2}$}

Existence of feedback regulation reflecting metabolic resistance in the starch biosynthesis pathway at high $\mathrm{CO}_{2}$ concentration: Increasing $\mathrm{CO}_{2}$ concentrations from $4 \times 10^{-8}$ to $4 \times 10^{-4} \mathrm{mM}$, we found a convex point of the metabolic regulation, or, the state at which the behaviour of the system (i.e., metabolite concentrations and fluxes) is converse (from positive deviation with respect to the reference point to the negative deviation, or vice versa). At low $\mathrm{CO}_{2}$ concentrations, metabolite concentrations and fluxes increased with $\mathrm{CO}_{2}$ elevation, but at a certain threshold of increased $\mathrm{CO}_{2}$ concentration $\left(4 \times 10^{-5} \mathrm{mM}\right.$ in this model), the system adjusted those entities antagonistically to the given amount of $\mathrm{CO}_{2}$ (as seen in a change from red to green in the heat map plot, Figure 3). This occurrence may explain the variation in the experimental results that come from testing the effect of $\mathrm{CO}_{2}$ elevation on starch biosynthesis, in which the testing range of $\mathrm{CO}_{2}$ abundance is critical [44]. Moreover, the feedback regulation suggested by the redirection of the metabolic adjustment under high $\mathrm{CO}_{2}$ concentrations may imply the metabolic response to the unlimited amount of $\mathrm{CO}_{2}$ that might harm or cause stress to plants $[45,46]$.

Triose phosphate (GAP and DHAP) and fructose-1, 6-bisphosphate (FBP) are key intermediates in starch biosynthesis: GAP, DHAP, and FBP concentrations (Figure 3a) and fluxes (V5, V6, V31, and V31a; Figure 3b)) are highly altered under $\mathrm{CO}_{2}$ elevation when compared to the other metabolic components. The significance of these metabolite alterations is more obvious in starch degradation and sucrose synthesis pathways than in the Calvin cycle, which contains many metabolites directly affected by the increased $\mathrm{CO}_{2}$, such as RuBP and Ru5P. The demonstrated sensitivities of the triose phosphate and FBP to metabolic perturbations suggest the importance of these intermediate metabolites in mediating the system normality and driving a metabolic response, causing a distorted plant physiology.

Leaf starch accumulation is highly influenced by $\mathrm{CO}_{2}$ concentration while tuber starch shows subtle effect: Interestingly, this study finds an asymmetrical effect of $\mathrm{CO}_{2}$ concentration on starch content in the leaf and tuber: a greater alteration in the quantity of leaf starch over that of tuber starch is observed in Figure 3. The overall metabolic alteration, as displayed in the heat map (Figure 3), indicates that $\mathrm{CO}_{2}$ availability likely affects metabolites in the carbonfixation cycle, which react (almost) directly with $\mathrm{CO}_{2}$. The result of the varied $\mathrm{CO}_{2}$ concentrations still obviously appears in the quantity of subsequent products of the Calvin cycle, such as leaf starch and triose sugars. The remaining metabolites derived from transitory starch show substantially less sensitivity to $\mathrm{CO}_{2}$ abundance, including tuber starch.

Starch biosynthesis is a sink-process-limited system: Many experimental data have implied that starch production in plants is limited by biochemical processes in non-photosynthetic or sink cells (e.g., tubers and endosperm) (Dupont, 2008; Sakulsingharoj et al., 2004). However, it is difficult to find a demonstration of alteration of metabolic intermediates under a particular perturbation to confirm this hypothesis. Plotting the alteration of metabolic intermediates across cells (Figure 3) allowed us to observe the robustness of the starch synthesis process in the tuber upon $\mathrm{CO}_{2}$ elevation. The plot indicates significant metabolic changes in leaf metabolism when compared to the tuber. Such metabolic changes in the tuber are re-plotted in Figure 4 , where the alteration of constituent metabolites can be observed. 

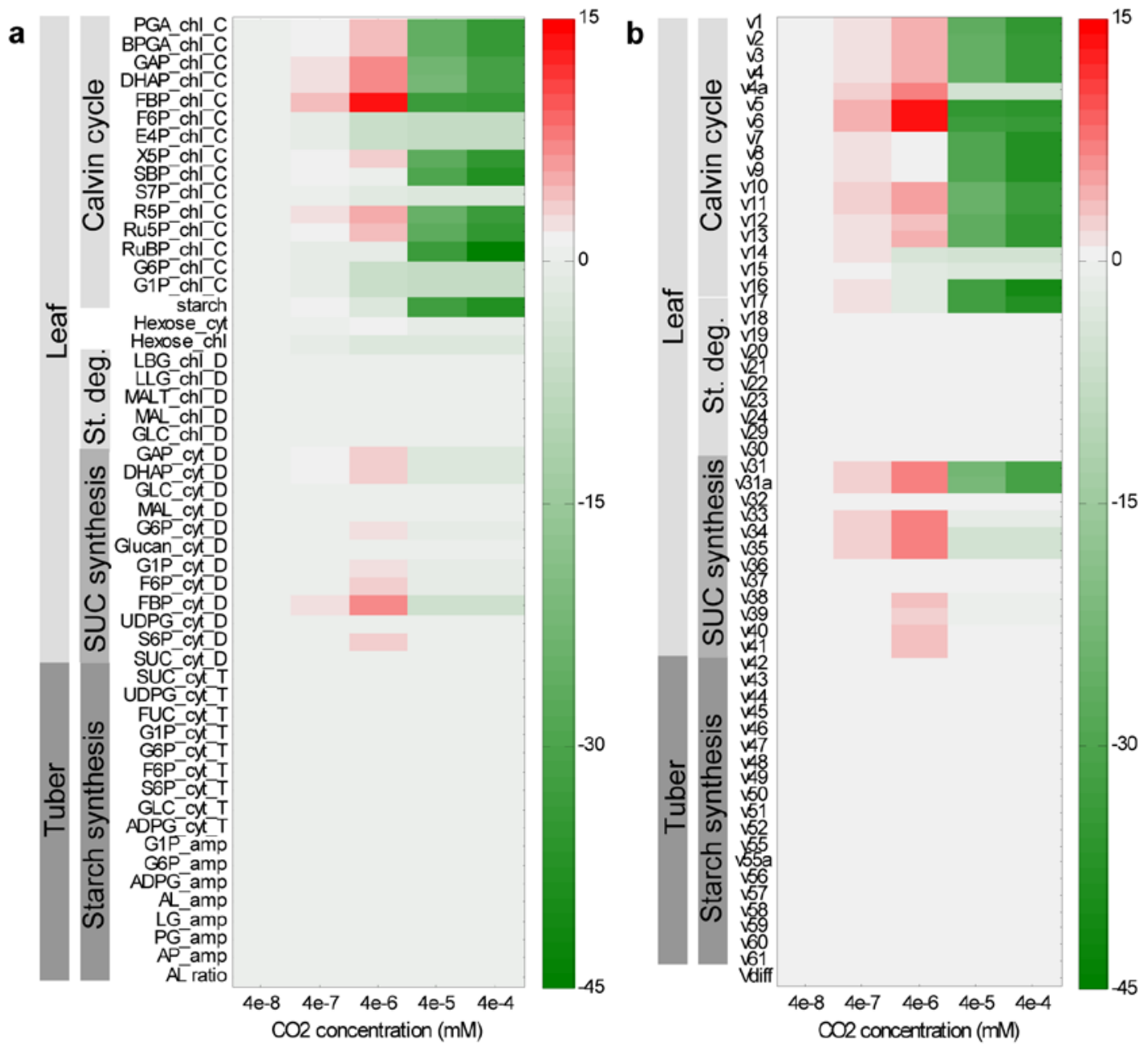

Figure 3: Changes in metabolism inside plant cells under in silico $\mathrm{CO}_{2}$ elevation. Heat map plot shows the metabolic changes inside plant cells under in silico $\mathrm{CO}_{2}$ elevation: (a) metabolite concentrations and (b) fluxes through enzymes in the starch biosynthesis pathway. The colour intensity and tones represent magnitude and direction of metabolic changes, respectively; magnitude of change ranging from grey (reference) to red (more deviation from reference in positive direction) or green (more deviation from reference in negative direction). Changes from red to green as shown in the figure indicate the presence of a convex point, or a state at which metabolism conversely changes the direction of biochemical reactions within the pathway.

As metabolism in the tuber is comparatively less affected by increased $\mathrm{CO}_{2}$ concentrations, the hypothesis still persists. Thus, we performed further tests to assure the restriction of the starch biosynthesis by the sink biochemical processes using in silico experiments.

First, we tested whether starch biosynthesis is limited by the transportation of leaf sucrose (SUC_cyt_D) to the tuber. The diffusion coefficient (Diff_coeff) was increased 10-fold to investigate the effect of an increased transportation rate on the tuber starch content (amylose (AL_amp) + amylopectin (AP_amp)). The result showed no change in the amount of produced starch, indicating no transportation restriction between the leaf and tuber (Figure 5).

Furthermore, we questioned the limitation of starch biosynthesis on the amount of sucrose. Failure of the last test on phloem transportation may be a result of a low concentration of sucrose in the leaf (SUC_ cyt_D), a product of starch degradation, causing a limitation in the concentration gradient instead of the rate constant. Moreover, it might be argued that a model of transportation in the phloem is very simple and might be far from plant nature. The test of the amount of sucrose confining tuber-starch content was performed by increasing the initial amount of sucrose in the tuber (SUC_cyt_T). The obtained result is similar to the case of increasing Diff_coeff, yet the starch content herein is slightly higher (1\%; Figure 5). The result therefore indicates that the carbon supply in the form of sucrose only partially restricts tuber starch formation.

Transportation of carbon to amyloplast limits the starch synthesis in the tuber: Regarding the relative metabolic changes within a tuber (Figure 4), the majority of cytosolic metabolite levels are highly affected by $\mathrm{CO}_{2}$ elevation, while all plastidic metabolite levels show inferior alteration. This led to the hypothesis that the carbon supply to amyloplast limits tuber starch synthesis. This thought is promising, as we observed unchanged transport fluxes (V55 and V55a; Figure $4 \mathrm{~b}$ ) of the carbon supply while there is a higher availability of carbon substrates in the cytosol (G6P_cyt_T and ADPG_cyt_T; Figure $4 a)$. We simulated the in silico over-expression of glucose-6-phosphate transporter (G6P/Pi in Figure 2) to examine whether the carbon supply across plastidic membrane restrains starch production in the tuber. Through increasing the rate of G6P transportation to amyloplast 


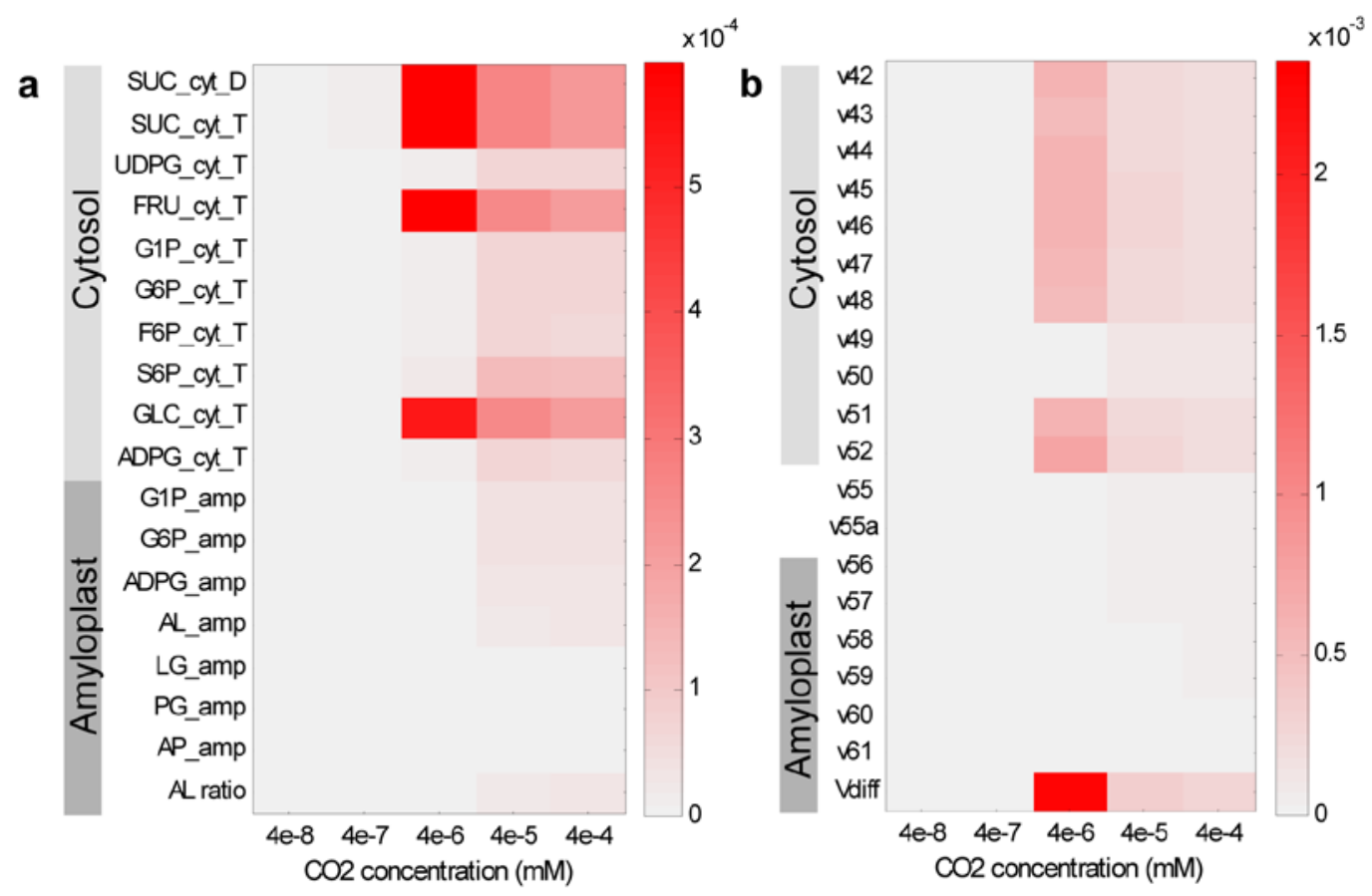

Figure 4: Changes in metabolism inside the tuber cell under in silico $\mathrm{CO}_{2}$ elevation. Heat map plotting shows the metabolic changes inside the tuber cell under in silico $\mathrm{CO}_{2}$ elevation: (a) metabolite concentrations and (b) fluxes through enzymes in the starch biosynthesis pathway. The colour intensity and tones represent magnitude and direction of metabolic changes, respectively, with the magnitude of change ranging from grey (reference) to red (more deviation from the reference in positive direction).

(V55), tuber-starch content is dramatically increased (58\%; Figure 5). The result strongly suggests that the carbon supply via sugar phosphate translocator is a limiting step in starch biosynthesis. The suggestion corresponds to the result of the independent study on the Metabolic Control Analysis (MCA) of starch biosynthesis in the tubers [6].

Starch synthesis in the tuber is restricted to the abundance of energy molecules (ATP): Regarding the relative metabolic changes within a tuber (Figure 4), the majority of cytosolic metabolite levels are highly affected by $\mathrm{CO}_{2}$ elevation, while all plastidic metabolite levels show inferior alteration. This led to the hypothesis that the carbon

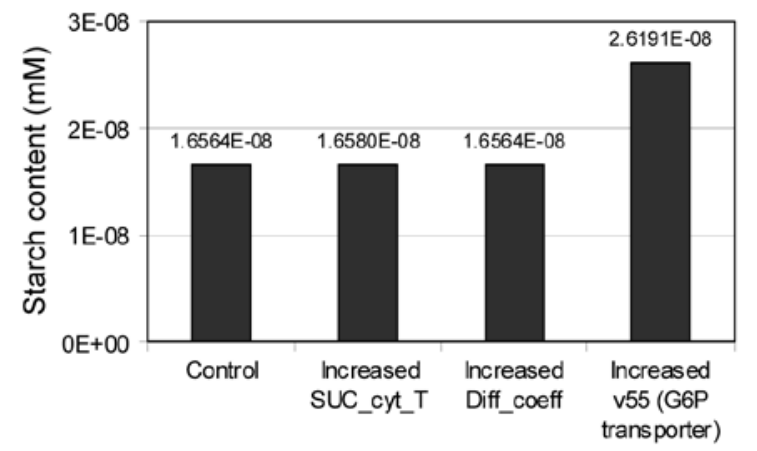

\section{Metabolic perturbations}

Figure 5: The effect of metabolic perturbations on the starch content. Simulating the starch content under certain metabolic perturbations: increased sucrose concentration in the tuber (SUC_cyt_T), increased transportation rate from the leaf cell to the tuber cell (Diff coeff), and increased transportation rate of G6P transporter in the tuber (v55); the relevant parameters to the case of perturbations were increased 10 -fold. supply to amyloplast limits tuber starch synthesis. This thought is promising, as we observed unchanged transport fluxes (V55 and V55a; Figure $4 \mathrm{~b}$ ) of the carbon supply while there is a higher availability of carbon substrates in the cytosol (G6P_cyt_T and ADPG_cyt_T; Figure 4a). We simulated the in silico over-expression of glucose-6-phosphate transporter (G6P/Pi in Figure 2) to examine whether the carbon supply across plastidic membrane restrains starch production in the tuber. Through increasing the rate of G6P transportation to amyloplast (V55), tuber-starch content is dramatically increased (58\%; Figure 5). The result strongly suggests that the carbon supply via sugar phosphate translocator is a limiting step in starch biosynthesis. The suggestion corresponds to the result of the independent study on the Metabolic Control Analysis (MCA) of starch biosynthesis in the tubers [6].

To reinforce the idea that energy substrates restrict the amount of starch production in the tuber, the in silico experiment was performed to estimate the tuber starch content with an increased ATP initial concentration (ATP_T at $\mathrm{t}=0 \mathrm{~s}$ ) at a certain amount of $\mathrm{CO}_{2}$. As seen in Figure 8, tuber-starch content increases with the quantity of ATP present in the tuber, supporting the restriction of such substrates on starch biosynthesis.

\section{Changes in metabolic flux distribution inferring a formal or preferential metabolic path responsible for starch production under high $\mathrm{CO}_{2}$ availability}

This section aims to provide the biological information interpreted from the metabolic flux distribution in the starch biosynthesis pathway simulated under varied $\mathrm{CO}_{2}$ concentrations. Together with simulated metabolite concentrations, the formal metabolic route-defined as a preferential pathway in plants used for assimilating carbon under nonlimited carbon supply-can be inferred by following the simulated flux distribution. 
Flux distribution in the Calvin cycle (labelled in grey in Figure 2):

\section{- Triose phosphate highly transports to the cytosol}

Basically, $\mathrm{CO}_{2}$ is taken from the atmosphere to initiate the Calvin cycle by reacting with ribulose-bisphosphate (RuBP). The products of this reaction are, in turn, a substrate for generating RuBP to supply the next round of the carbon fixation reaction. Availability of the triose phosphate (GAP and DHAP; synthesised in the Calvin cycle) in chloroplasts is crucial to proceed the carbon fixation cycle, yet this three-carbon sugar molecule is diverted to the cytosol for other uses. Therefore, the flux distribution underlying the availability of triose phosphate (GAP and DHAP) in chloroplasts was first determined. The compared flux distribution of triose phosphate (V5-to the Calvin cycle, and V31 and V31a-to cytosol) implies that the produced three-carbon sugar is mainly exported to the cytosol, while a relatively small amount is retained for use in the carbon fixation cycle (Figure 9a).

- Chloroplastic fructose-6-bisphosphate (F6P) is mostly used in transitory starch formation rather than in Calvin cycle recirculation

F6P in the Calvin cycle is derived from triose phosphate as an intermediate for generating RuBP and as a precursor for polymerising leaf starch. Flux distributions relating to F6P (V7 and V14) indicate that F6P is preferentially converted to G6P, an intermediate to form transitory starch (Figure S1a).

Flux distribution in leaf-starch breakdown and sucrose synthesis pathways (labelled in yellow in Figure 2):

- Maltotriose (MALT) is a major residue resulting from transitory starch breakdown

Transitory starch, which forms during the day, is broken-down into many smaller molecules via different paths. These small residues,

a

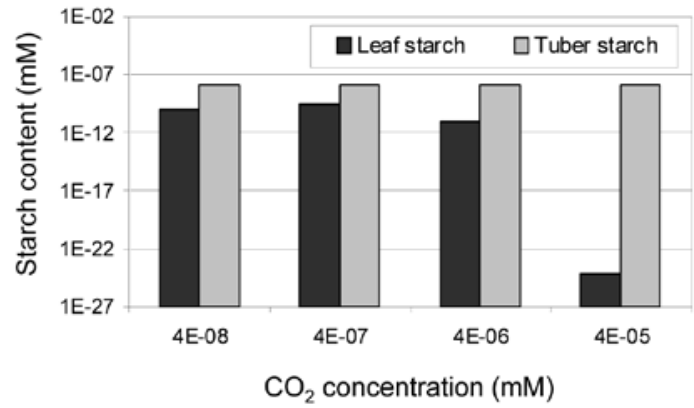

b

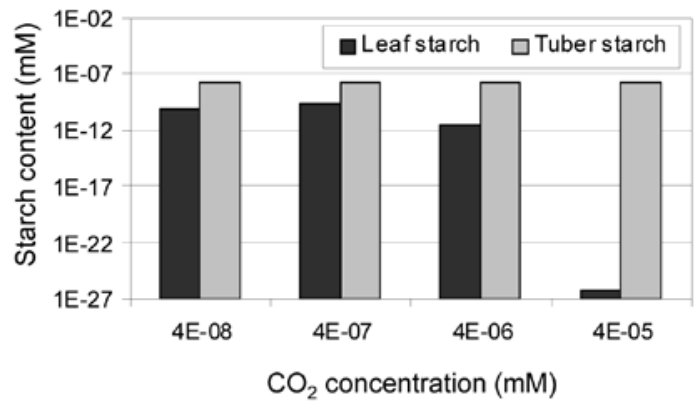

Figure 6: Model simulation under different assumptions on currency metabolites. The estimated starch content under $\mathrm{CO}_{2}$ elevation by assuming (a) excess and (b) non-excess concentrations of current metabolites; black-leaf starch, and grey-tuber starch. including G1P, MALT, and maltose (MAL), are the initiators for production of leaf sucrose (SUC_cyt_D), which is subsequently translocated for use as a carbon substrate in the tuber. To examine the plant's favourite route for starch degradation, flux partitions relevant to the long linear glucan (LLG) were compared. Figure $9 \mathrm{~b}$ exhibits significantly greater degradation fluxes from LLG to MALT (V21, V22, and V23) than from LLG to the other residues (V19 and V20), suggesting that it is the formal metabolic pathway in starch degradation under high $\mathrm{CO}_{2}$ concentration.

- Leaf sucrose (SUC_cyt_D) is mainly formed from cytosolic glucose derivatives rather than from triose phosphate

Regarding the metabolic pathway described in Figure 2, sucrose in the leaf is derived either from the imported triose phosphate or the glucose and maltose (from starch degradation). Metabolic fluxes of those possibilities (Figure $9 \mathrm{c}$ and $\mathrm{S1c}$ ) demonstrate that triose phosphate tends to accumulate in the leaf cytosol or be utilised in other pathways, such as glycolysis, whereas glucose (and maltose) derivatives are responsible for sucrose synthesis.

Flux distribution in starch synthesis pathway in the tuber (labelled in blue in Figure 2):

- UDP glucose (UDPG_cyt_T) is an active intermediate functioning to derive a carbon substrate (G6P and ADPG) for tuber-starch formation

Complicated metabolic reactions take place in the tuber to transform the transported sucrose (SUC_cyt_T) into a carbon skeleton for supplying starch synthesis in the amyloplast. As seen in Figure 9d, the formation of G6P through UDPG (V42) is a dominant pathway over breaking sucrose down to glucose (GLC_cyt_T) and fructose (FUC_cyt_T)(V45).

- Glucose-6-phosphate (G6P_cyt_T) is a main carbon supply for tuber-starch formation

To address the question of which carbon substrate is preferred for starch formation, we compared the metabolic fluxes from G1P to G6P (V44) and G1P to ADPG (V52; Figure 9e). The result shows that G6P is likely to be a main carbon substrate passing through the amyloplastic membrane for supplying starch synthesis, though G6P must be reconverted to G1P and then ADPG before being polymerised (Figure $9 \mathrm{e}$ ). This finding is also supported by the following investigation on a major source of ADPG available in the tuber amyloplast (see next section for more detail).

- Amyloplastic ADP glucose (ADPG_amp), which is a precursor for carbon-chain polymerisation in starch biosynthesis, is mostly obtained from G1P conversion in the amyloplast

One of the most discussed issues in starch biosynthesis research is how cytosolic ADPG transports into amyloplast, because the ADPG transporter is not present, or at least has not yet been identified in all plant species (except some crops (Bowsher et al., 2007)). Our model deals with such a problem by assuming that cytosolic ADPG moves across the amyloplastic membrane via the G6P transporter. We then examined whether or not the transported ADPG is a main input of ADPG used for starch synthesis in the amyloplast. Comparing the two input fluxes of amyloplastic ADPG-transported from the cytosol (V55a) and converted from the transported G6P (V57)-revealed that the abundance of ADPG in the amyloplast greatly depends on the availability of the transported G6P (Figure 9f): ADPG conversion flux 
Citation: Saithong T, Meechai A, Cheevadhanarak S, Bhumiratana S (2012) A Formal Path Inference of Starch Biosynthesis via Mathematical Modelling of Metabolic Changes in Excess $\mathrm{CO}_{2}$. J Comput Sci Syst Biol 5: 024-037. doi:10.4172/jcsb.1000087

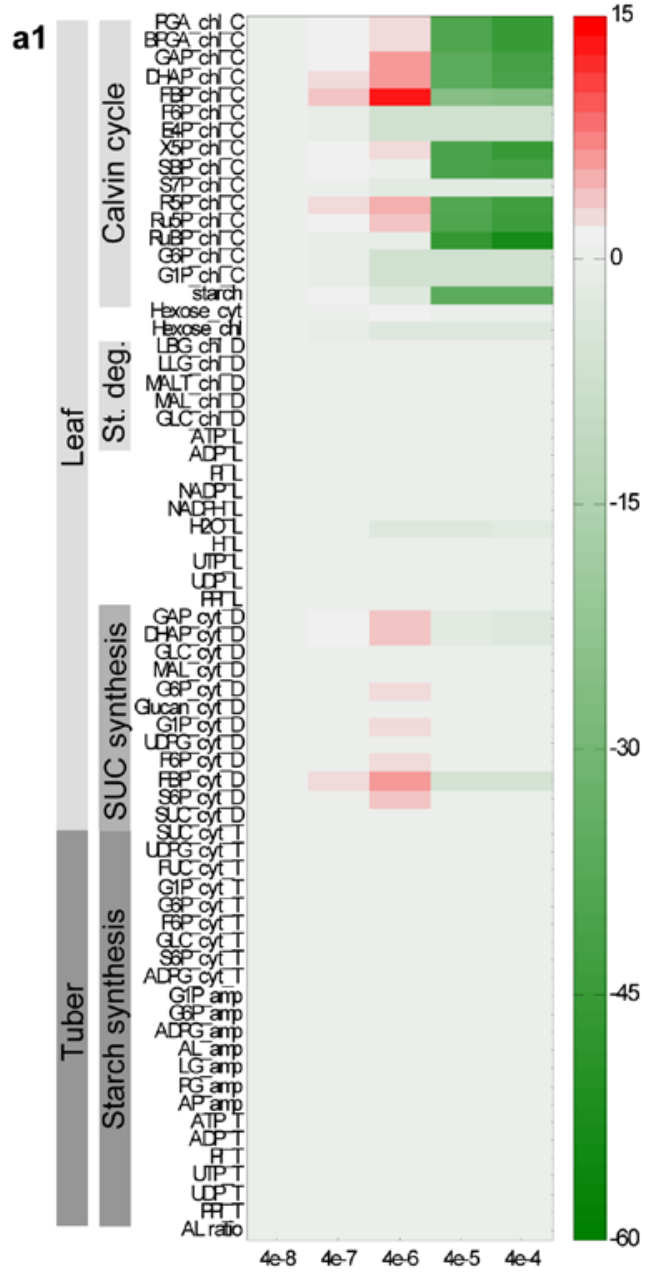

$\mathrm{CO}_{2}$ concentration (mM) b1

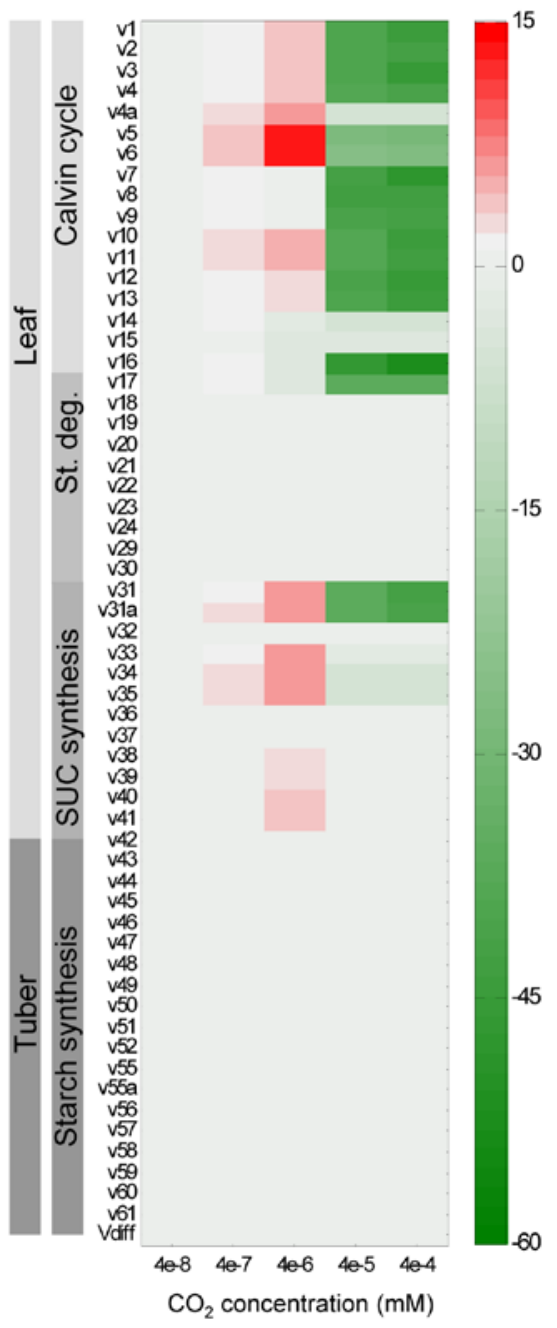

a2

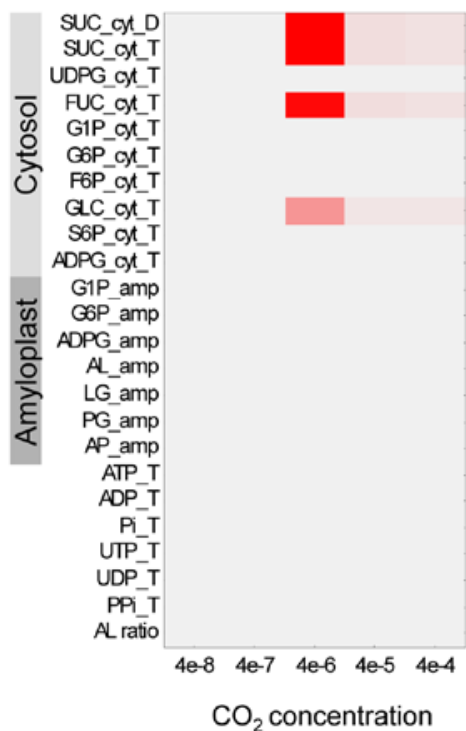

$\times 10^{-3}$

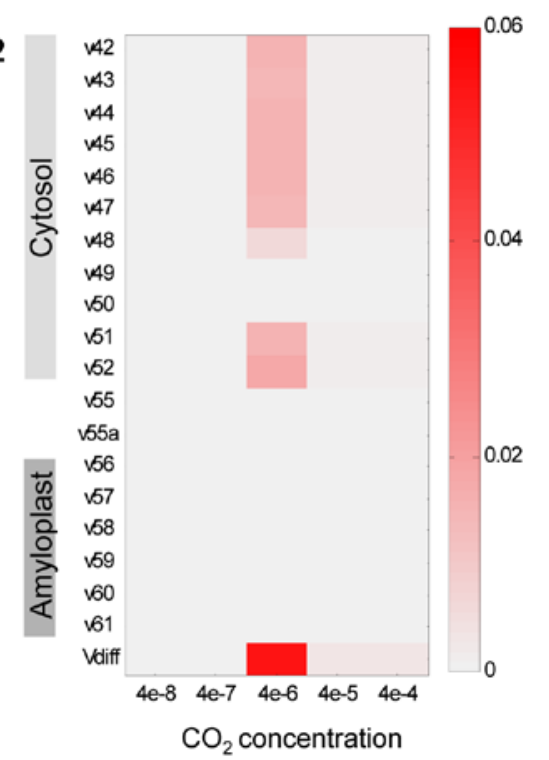

Figure 7: Changes in metabolism under in silico $\mathrm{CO}_{2}$ elevation (currency metabolites are not in excess.). Heat map plotting shows the metabolic changes in (1i) plant cells and (2) tuber cell under in silico $\mathrm{CO}_{2}$ elevation by assuming non-excess concentrations of currency metabolites: (a) the metabolite concentrations and (b) the fluxes through enzymes in the starch biosynthesis pathway. The colour intensity and tones represent magnitude and direction of metabolic changes, respectively, with the magnitude of change ranging from grey (reference) to red (more deviation from the reference in positive direction) or green (more deviation from the reference in negative direction). 


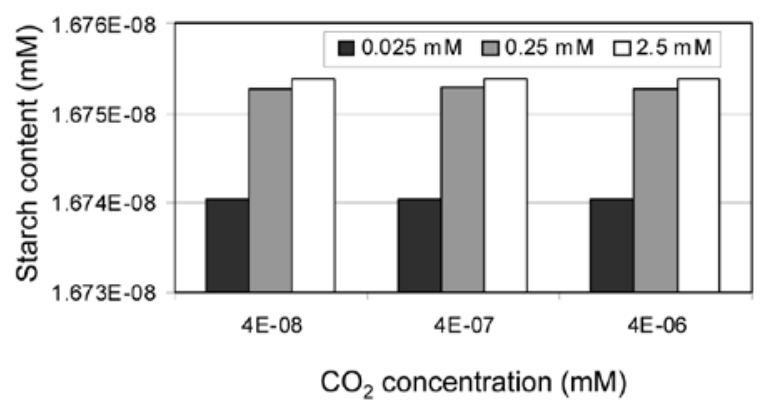

Figure 8: The effect of tuber ATP elevation on the starch content. Simulating starch content while increasing the initial concentration of the tuber ATP (ATP_T; 0.025 mM-black, $0.25 \mathrm{mM}$-grey, and $2.5 \mathrm{mM}$-white) at a certain $\mathrm{CO}_{2}$ concentrations.

from G6P is approximately four times higher than its transportation flux.

- Increasing the availability of the carbon substrate alters the property of starch by raising the amylose-to-amylopectin ratio (AL/AP) or percentage of amylose

Despite a lack of knowledge on how amylose and amylopectin form a starch granule, we have learnt the effect of $\mathrm{CO}_{2}$ elevation on starch properties through the amylose-to-amylopectin ratio. Amyloserich starch was predicted under excess carbon-substrate conditions by examining fluxes underlying amylose (V58) and amylopectin (V59) production, which are partitioned by the efficiency of the granulebound (GBSS) and soluble (SS) starch synthase enzymes (Figure 9g).

Taking all these results together, we can propose the formal metabolic path underlying the different starch phenotypes in plants. Figure 10 illustrates the metabolic route having a high flux distribution under a non-constrained carbon supply (marked with red arrows) that presumably plays a main role in converting the (excess) incoming carbon substrate to starch. To summarise, the fixed one-carbon molecule $\left(\mathrm{CO}_{2}\right)$ reacts with the five-carbon molecule $(\mathrm{RuBP})$ to form a three-carbon sugar molecule (triose phosphate) that is preferentially exported to accumulate in the cytosol. While only small amounts of metabolites in the Calvin cycle are required to sustain the carbon fixation cycle, the rest are used in the formation of transitory starch. Subsequently, the transitory starch is decomposed into various small carbon molecules, especially glucose and maltose that are the main substrates for synthesising sucrose in the cytosol of the leaf cell. Once sucrose is transported to a sink cell, it is broken-down mostly to UDPG instead of GLC and FUC. Such produced UDPG is selectively applied for G6P generation, a preferred carbon substrate taken into the amyloplast for starch biosynthesis. Accordingly, overt variations in starch phenotype, either quality- or quantity-based, are presumably caused by an alteration in one of the components (processes) within this route.

\section{Discussion and Conclusions}

This work intends to provide insight into metabolic regulation of the starch biosynthesis pathway in C3-plants, storing starch in a tuber. Perhaps, it might be established as a model for potato starch biosynthesis, as more than half of the reactions in the modelled pathway were collected from potato literature and the majority of the employed kinetic parameters (139/294 47\%) were suggested by the measurements in potato and its previous models. Mathematical modelling was used as a tool to incorporate known information, and to allow us to observe the system and analyse the obtained data systematically. Our results are useful in supporting the intuitive thoughts hypothesised from the experimental data, leading to increased comprehension of the studied network in multiple ways.

First, we have shown that there is a feedback regulation in carbon metabolism at high $\mathrm{CO}_{2}$ concentrations. Such an abundance of $\mathrm{CO}_{2}$ may introduce stress to plants and cause biological damage, as reported in $[45,46]$. The down-regulation of metabolites in the carbon fixation cycle (Figure 3) might be a metabolic defence mechanism of plants, responding to prevent highly excessive $\mathrm{CO}_{2}$.

Second, our results have highlighted the significance of triose phosphate (both GAP and DHAP) and FBP in the starch biosynthesis pathway. Their sensitivity to metabolic perturbation was observed from an apparently great alteration in their concentrations under $\mathrm{CO}_{2}$ concentrations. Triose phosphate and FBP, in fact, play pivotal roles as key metabolites in various metabolic pathways where they are present, especially in central metabolism, such as glycolysis [10]. Reasons behind their presence might be as follows: (1) triose phosphate is an early product of carbon fixation that can be localised to the cytosol and be utilised in diverse manners; (2) triose phosphate as well as FBP are easily transformed or converted to many other metabolites, which are an important property of a key intermediate.

Third, this study has demonstrated a complicated regulation network inside the starch biosynthesis pathway that might be hard to exhibit through real-time measurements. Experiments attempting to investigate starch content under varying $\mathrm{CO}_{2}$ concentrations have reported inconsistent results, and the final solution regarding the relationship between the two entities remains controversial dependent of experimental conditions $[18,23,24]$. Such conflicted experimental data are mostly described by the variation in experimental conditions, including tested plant species, plant cultivar, and chemical concentrations (i.e., metabolite and substrate). Our finding of an asymmetrical effect of $\mathrm{CO}_{2}$ on the transitory starch in the leaf and permanent starch in the tuber might be another explanation of the existing discrepancy in the literature and would be a point of concern for the next experiment.

Fourth, we have described evidence supporting a sink-limited hypothesis addressed on the regulation of starch biosynthesis (Figure 3,4). Plastidic ADP-glucose pyrophosphorylase (AGPase) is the enzyme that most likely controls starch yield in plants [16,19-21]. It is, therefore, a favoured target for metabolic engineering by aiming at producing a high-starch-content phenotype $[16,20]$. However, recent data shows that enhancing AGPase activity generally does not result in greater starch content per gram fresh weight (reviewed in Smith, 2008). This failure may be caused by a strong allosteric effect, or a complication of AGPase enzyme composed of several subunits. As a consequence, the main focus of many current studies, including ours, has changed toward substrate limitation [14,17,18,22-24] instead of the restricted activity of the enzyme. Our in silico test on the carbonsubstrate supply suggests that G6P transport to the amyloplast is a system bottleneck that causes a sink limitation in starch production (Figure 3-5). Furthermore, the test on the energy-substrate (ATP) supply also points out its restriction to the yield of starch production (Figure 8). The related experimental data implying substrate-limitation could be found in $[17,18]$ (energy or adenylate substrates) and $[23,24]$ 
Citation: Saithong T, Meechai A, Cheevadhanarak S, Bhumiratana S (2012) A Formal Path Inference of Starch Biosynthesis via Mathematical Modelling of Metabolic Changes in Excess $\mathrm{CO}_{2}$. J Comput Sci Syst Biol 5: 024-037. doi:10.4172/jcsb.1000087

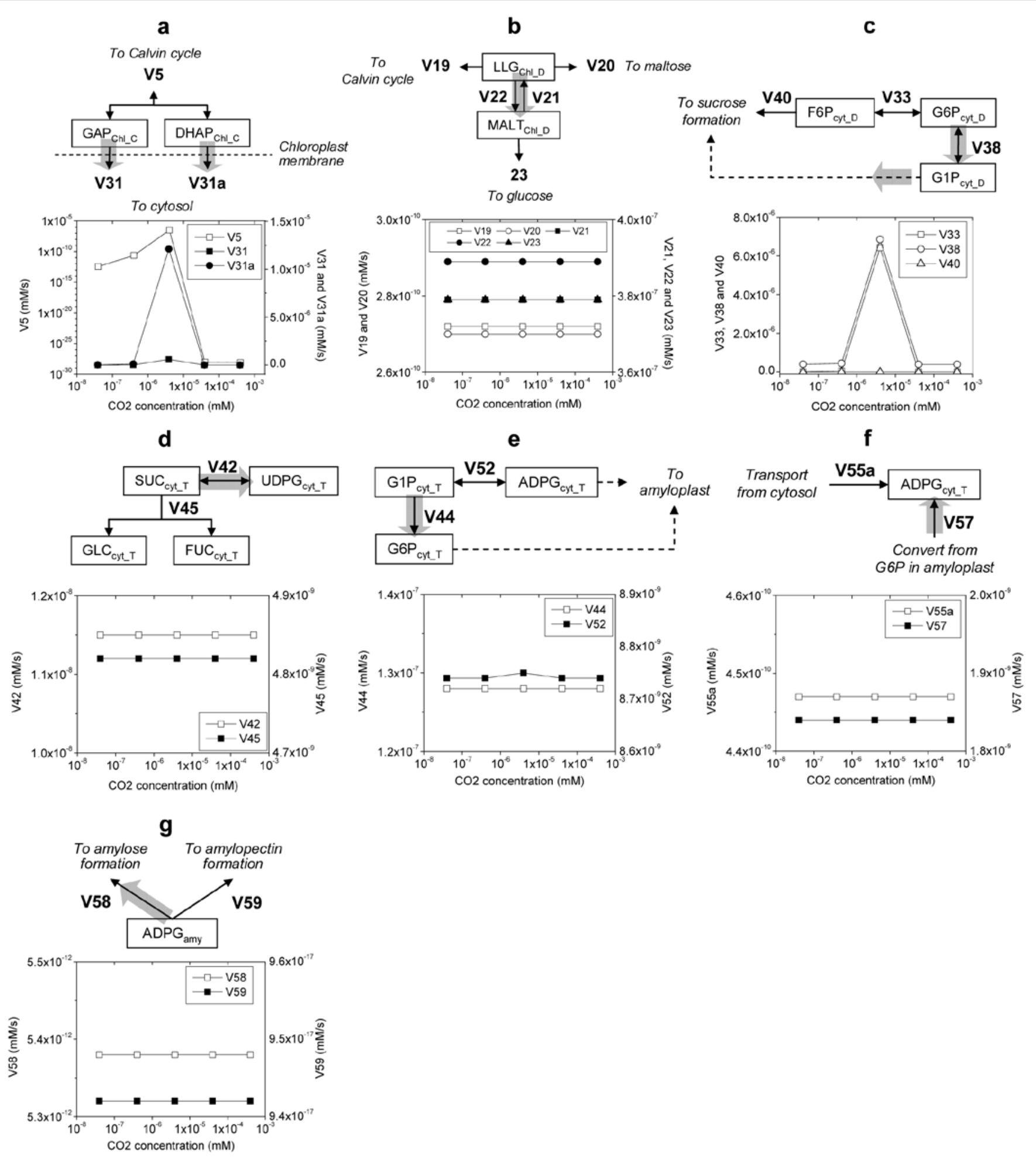

Figure 9: Simulation of flux distribution under $\mathrm{CO}_{2}$ elevation. Simulating flux distribution at any key branch-points within the network infers a formal metabolic route response in the different starch formation under $\mathrm{CO}_{2}$ elevation (grey arrows): fluxes through (a) aldolase (V5) and triose-phosphate transporter (V31-GAP and V31a-DHAP); (b) starch phosphorylase (V19), beta-amylase (V20), disproportional enzyme (V21 and V23), and alpha-amylase (V22); (c) phosphoglucomutase (V33), phosphoglucoisomerase (V38) and sucrose phosphate synthase (V40); (d) sucrose synthase (V42) and invertase (V45); (e) tuber phosphoglucomutase (V44) and cytosolic ADPG pyrophosphorylase (V52); (f) G6P transporter (V55a-ADPG) and plastidic ADPG pyrophosphorylase (V57); (g) granule-bound starch synthase (V58) and starch synthase (V59). $\mathrm{CO}_{2}$ concentrations (x-axis) and fluxes ( $\mathrm{y}$-axis) are simulated in $\mathrm{mM}$ and $\mathrm{mM} / \mathrm{s}$, respectively. Clear symbols are results plotted against the left $y$-axis, while filled symbols are those plotted against the right y-axis. Grey arrows superimposed on each cascade represent the preferential metabolic route of plants, suggested by flux distribution (summarised in Figure 10). 


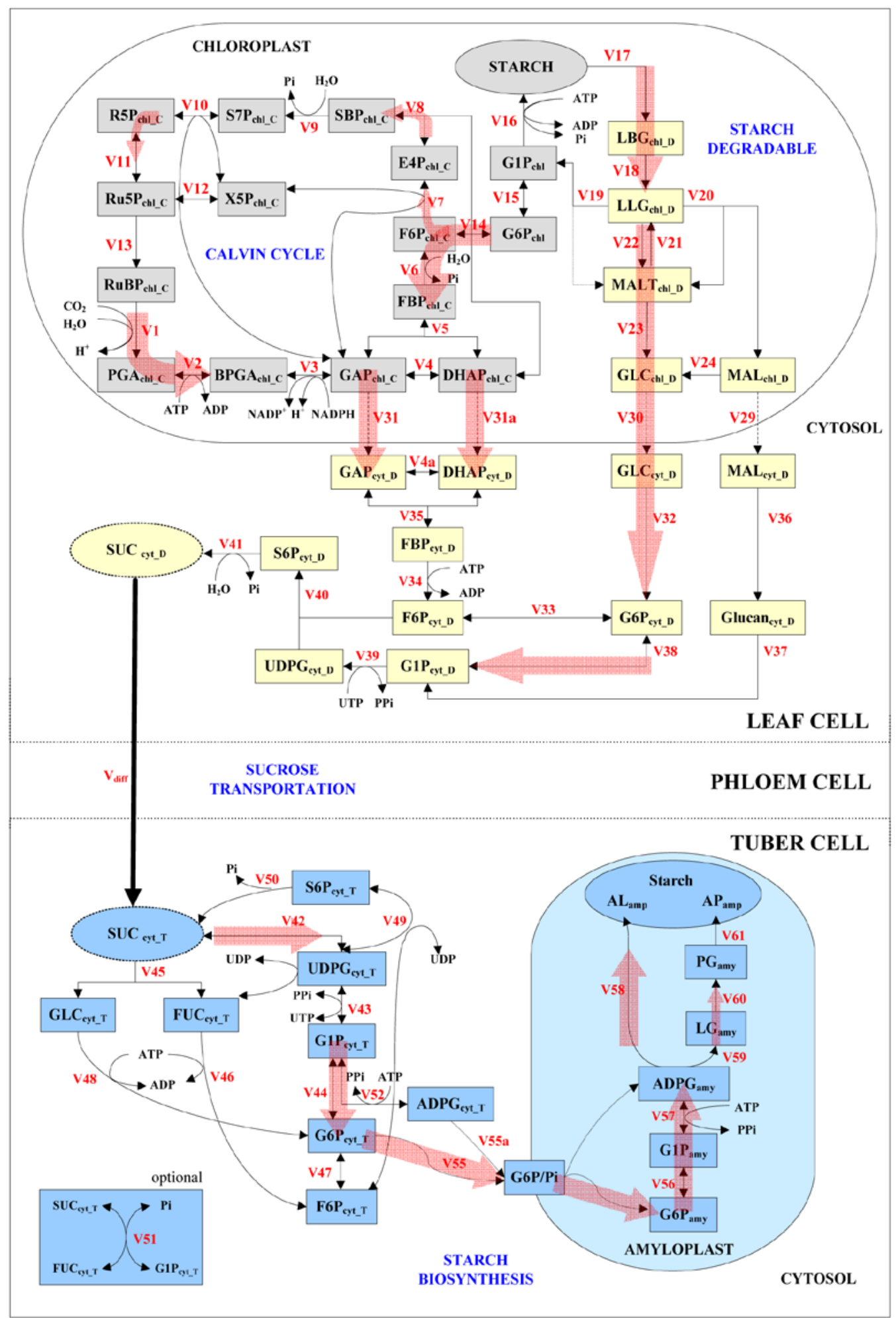

Figure 10: The proposed formal metabolic route response for starch production under high concentration of $\mathrm{CO}_{2}$. The scheme illustrates the potential, formal metabolic route for starch production under high concentration of $\mathrm{CO}_{2}$ (red arrows, drawn corresponding to the grey arrows in Figure 9 ) that was proposed according to the simulations and analysis in Figure 9.

(carbon substrates), for example, among which Zhang et al. introduced the most sophisticated regulation relying on the co-limitation of carbon and energy substrates on starch biosynthesis [18].
Apart from starch yield, elevating $\mathrm{CO}_{2}$ concentration affects the AL/AP-ratio-related properties of starch. The predicted highamylose content is understandable when you take into account the 
different affinity of the two isoforms of starch synthase enzymes. GBSS, functioning in amylose production, has a lower affinity to the substrate than SS, whose efficiency dictates the amount of amylopectin. Therefore, under an excess of substrate, GBSS efficiency is expected to highly increase over SS, leading to the increased amylose percentage in the produced starch [47].

Integrating all analysed results, we have finally proposed a formal metabolic route, potentially employable in C3-plant carbon anabolism to produce starch. The proposed pathway has been concluded from our understanding of the regulation of starch biosynthesis based on flux distributions and metabolite concentrations (simulated under $\mathrm{CO}_{2}$ elevation), and background knowledge. With respect to the initial datasets used to build the model, our results have made the previous biological implications less abstract by providing quantitative comparisons and testing hypotheses numerically. The insight into metabolic regulation of starch biosynthesis presented here (i.e., metabolic responses and formal metabolic route) is expected to facilitate biologists generating a new hypothesis and designing the next testable experiment.

A mathematical model is only as valid as its defined assumptions. The constructed model is really a first draft of an ambitious model representing the whole starch biosynthesis pathway involving elaborated metabolic networks in multi-cells. Various assumptions (e.g. light/dark cycle) are required to set the validity of the model under limitations of data and modelling techniques. Sometimes, less realistic assumption is needed to make modelling of the system feasible. Despite the concerns on model simplification against biological complexity, it has been shown many times that the highly simplified models are useful for investigating the complex systems, especially at the early stage such as circadian clock $[48,49]$. On the other hand, incorporating complexity into the model to imitate real situations without certainty (or clear knowledge) may result in model sloppiness [50].

In this work, the model of the starch biosynthesis pathway that spans source-sink cells gives good simulations and reasonable predictions in the system regulation according to its simplifications. Nonetheless, to allow the current model to investigate more sophisticated regulation in the studied pathway, it needs to be improved to account for some of the assumptions. Particularly, a more realistic mechanism should be used to replace the transport through the phloem, and adenylate compound translocation should be revisited. The next model might be extended to include diurnal regulation and sucrose signalling, whose effects on starch biosynthesis have been extensively discussed [25,51,52]. As plant starch is one of the largest food sources for all life on Earth and the mechanism underlying starch production is varied among species, our hope is that these findings will accelerate this research field in the future.

\section{Authors' Contributions}

TS constructed multi-cellular model of starch biosynthesis, performed all of analyses, and wrote the manuscript. AM contributed in discussion and manuscrip revision. SC and SB are funding consultants and shared ideas on this research in principle. All authors have read and approved the final manuscript.

\section{Acknowledgements}

The authors would like to thank Piyaporn Saraboon, who helped us to develop a preliminary model of starch biosynthesis. We also thank Papapit Ingkasuwan and Chalotorn Liamwirat for useful discussion.

\section{References}

1. Smith AM (2001) The biosynthesis of starch granules. Biomacromolecules 2 : 335-341.
2. Martin C, Smith A (1995) Starch biosynthesis. Plant Cell 7: 971-985

3. Smith AM (2008) Prospects for increasing starch and sucrose yields for bioethanol production. Plant J 54: 546-558.

4. Burrell MM (2003) Starch: the need for improved quality or quantity-an overview. J Exp Bot 54: 451-456.

5. Zeeman S, Delatte T, Messerli G, Umhang M, Stettler M, et al. (2007) Starch breakdown: recent discoveries suggest distinct pathways and nove mechanisms. Funct Plant Biol 34: 465-473.

6. Saithong T, Meechai A, Chevadhanarak S, Bhumiratana S (2004) Metabolic control analysis of starch biosynthesis pathways in amyloplast organelle. Tha J Biotechnol 5: 23-33.

7. Junker BH (2004) Sucrose breakdown in the potato tuber [PhD Thesis] Potsdam: Universitit Potsdam.

8. Poolman MG, Fell DA, Thomas S (2000) Modelling photosynthesis and its control. J Exp Bot 51: 319-328.

9. Ball SG, van de Wal MHBJ, Visser RGF (1998) Progress in understanding the biosynthesis of amylose. Trends Plant Sci 3: 462-468.

10. Preiss J (1982) Regulation of the biosynthesis and degradation of starch. Annu Rev Plant Physiol 33: 451-454.

11. Ishizaki Y, Taniguchi H, Maruyama Y, Nakamura M (1983) Debranching enzymes of potato tubers (Solanum tuberosum L.). I. Purification and some properties of potato isoamylase. Agric Biol Chem 47: 771-779.

12. Ahn Y, Kim SH, Kim CY, Lee J, Kwak S, et al. (2010) Exogenous sucrose utilization and starch biosynthesis among sweetpotato cultivars. Carbohydr Res 345: 55-60.

13. Kunwong $U$, Chanvivattana $\mathrm{Y}$, Leelapon $\mathrm{O}$, Boonseng $\mathrm{O}$, Narangajavana J. Charcterisation of a gene highly expressed during early developmental stage of Cassava storage roots; 2008; Thailand.

14. Mohlmann T, Tjaden J, Henrichs G, Quick WP, Hausler R, et al. (1997) ADP glucose drives starch synthesis in isolated maize endosperm amyloplast characterization of starch synthesis and transporter properties across the amyloplast envelop. Biochem J 324: 503-509.

15. Zeeman SC, Pilling E, Tiessen A, Kato KL, Donald AM, et al. (2002) Starch synthesis in Arabidopsis. Granule synthesis, composition, and structure. Plant Physiol 129: 516-529.

16. Smidansky ED, Clancy M, Meyer FD, Lanning SP, Blake NK, et al. (2002) Enhanced ADP-glucose pyrophosphorylase activity in wheat endosperm increases seed yield. Proc Natl Acad Sci U S A 99: 1724-1729.

17. Tjaden J, Mohlmann T, Kampfenkel K, Henrichs G, Neuhaus E (1998) Altered plastidic ATP/ADP-transporter activity influences potato (Solanum tuberosum L.) tuber morphology, yield and composition of tuber starch. Plant J 16: 531 540.

18. Zhang L, Hausler RE, Greiten C,Hajirezaei MR, Haferkamp I, et al (2008) Overriding the co-limiting import of carbon and energy into tuber amyloplasts increases the starch content and yield of transgenic potato plants. Plant Biotechnol J 6: 453-464.

19. Lloyd JR, Springer F, Buleon A, Muller-Rober B, Willmitzer L, et al. (1999) The infuence of alterations in ADP-glucose pyrophosphorylase activities on starch structure and composition in potato tubers. Planta 209: 230-238.

20. Sakulsingharoj C, Choi S, Hwang S, Edwards GE, Bork J, et al. (2004) Engineering starch biosynthesis for increasing rice seed weight: the role of the cytoplasmic ADP-glucose pyrophosphorylase. Plant Sci 167: 1323-1333.

21. Ihemere U, Arias-Garzon D, Lawrence S, Sayre R (2006) Genetic modification of cassava for enhanced starch production. Plant Biotechnol J 4: 453-465.

22. Regierer B, Fernie B, Springer F, Perez M, Leisse A, et al. (2002) Starch content and yield increase as a result of altering adenylate pools in transgenic plants. Nat Biotechnol 20: 1256-1260.

23. Lawson T, Craigon J, Black CR, Colls JJ, Tulloch AM, et al. (2001) Effects of elevated carbon dioxide and ozone on the growth and yield of potato (Solanum tuberosum) grown in open-top chambers. Environment Pollution 111: 479-491.

24. Ning W, Park SN (1996) Doubling the $\mathrm{CO}_{2}$ concentration enhanced the activity of carbohydrate metabolism enzymes, source carbohydrate production, 
Citation: Saithong T, Meechai A, Cheevadhanarak S, Bhumiratana S (2012) A Formal Path Inference of Starch Biosynthesis via Mathematical Modelling of Metabolic Changes in Excess $\mathrm{CO}_{2}$. J Comput Sci Syst Biol 5: 024-037. doi:10.4172/jcsb.1000087

photoassimilate transport, and sink strength for opuntia ficus-indica. Plant Physiol 110: 893-902

25. Chiou T, Bush BR (1998) Sucrose is a signal molecule in assimilate partitioning. Plant Biol 95: 4784-4788.

26. de Oliveira Dal'Molin CG, Quek LE, Palferyman RW, Brumbley SM, Nielsen LK (2010) AraGEM, a genome-scale reconstruction of the primary metabolic network in Arabidopsis. Plant Physiol 152: 579-589.

27. Novère NL, Bornstein B, Broicher A, Courtot M, Donizelli M, et al. (2006) BioModels Database: a free, centralized database of curated, published, quantitative kinetic models of biochemical and cellular systems. Nucleic Acids Res 34: D689-D691.

28. Olivier BG, Snoep JL (2004) Web-based kinetic modelling using JWS Online. Bioinformatics 20: 2143-2144.

29. Poolman MG, Miguet L, Sweetlove LJ, Fell DA (2009) A genome-scale metabolic model of Arabiodpsis and some of its properties. Plant Physiol 151: 1570-1581.

30. Pettersson G, Ryde-Petterson U (1990) Model studied of the regulation of Calvin photosynthesis cycle by cytosolic metabolites. Biochem Biomed Acta 40: 723-732.

31. Farquhar GD, von Caemmerer S, Berry JA (2001) Models of photosynthesis. Plant Physiol 125: 42-45.

32. Rohwer MJ, Botha FC (2001) Analysis of sucrose accumulation in the sugar cane culm on the basis of in vitro kinetic data. Biochem J 358: 437-445.

33. Assmus HE (2005) Modelling the carbohydrate metabolism in potato tuber cells. [PhD Thesis]. Oxford: Oxford Brookes University.

34. Poolman MG (1999) Computer modeling applied to the Calvin cycle [PhD Thesis]. Oxford: Oxford Brookes University.

35. Schleucher J, Vanderveer PJ, Sharkey TD (1998) Export of carbon from chloroplasts at night. Plant Physiol 118: 1439-1445

36. Hahn BD (1984) A mathematical model of leaf carbon metabolism. Ann Bot 54: 325-339.

37. Geigenberger P (2003) Regulation of sucrose to starch conversion in growing potato tubers. J Exp Bot 54: 457-465.

38. Tiessen A, Hendriks JHM, Stitt M, Branscheid A, Gibon Y, et al. (2002) Starch synthesis in potato tubers is regulated by posttranslational redox modification of ADP-glucose pyrophosphorylase: a novel regulatory mechanism linking starch synthesis to the sucrose supply. Plant Cell 14: 2191-2213.

39. Millar AJ (2003) A suite of photoreceptors entrains the plant circadian clock. J Biol Rhythms 18: 217-226.

40. Locke JCW, Kozma-Bognar L, Gould PD, Feher B, Kevei E, et al. (2006) Experimental validation of a predicted feedback loop in the multi-oscillator clock of Arabidopsis thaliana. Mol Syst Biol doi:10.1038/msb4100102.
41. Wongpayak S (2006) Development of rigorous model for sucrose transportation process [Master Thesis]. Bangkok, Thailand: King Mongkut's University of Technology Thonburi.

42. Morgan AJ, Rhodes D (2002) Mathematical modeling of plant metabolic pathway. Metab Eng 4: 80-89.

43. Schomburg I, Chang A, Ebeling C, Gremse M, Heldt C, et al. (2004) BRENDA the enzyme database: updates and major new developments. Nucleic Acids Res 32: D431-D433.

44. Bunce J, Sicher RC (2003) Daily irradiance and feedback inhibition of photosynthesis at elevated carbon dioxide concentration in Brassica oleracea. Photosynthetica 4: 481-488.

45. Mott K (1999) Sensing of atmospheric CO2 by plants. Plant Cell Environ 13 731-737.

46. Shwartz M (2002) High carbon dioxide levels can retard plant growth, study reveals. California: Stanford University.

47. Smith A, Denyer K, Martin C (1997) The synthesis of the starch granule. Annu Rev Plant Physiol Plant Mol Biol 48: 67-87.

48. Goldbeter A (1995) A model for circadian oscillations in the Drosophila period protein (PER). Proc Biol Sci 261: 319-324.

49. Salazar JD, Saithong T, Brown PE, Foreman J, Locke JC, et al. (2009) Prediction of photoperiodic regulators from quantitative gene circuit models. Cell 139: 1170-1179.

50. Saithong T, Painter KJ, Millar AJ (2010) The contributions of interlocking loops and extensive nonlinearity to the properties of circadian clock models. PLoS ONE 5: e13867.

51. Blasing OE, Gibon Y, Gunther M, Hohne M, Morcuende R, et al. (2005) Sugars and circadian regulation make major contributions to the global regulation of diurnal gene expression in Arabidopsis. Plant Cell 17: 3257-3281.

52. Smith SM, Fulton DC, Chia T, Thorneycroft D, Chapple A, et al. (2004) Diurnal changes in the transcriptome encoding enzymes of starch metabolism provide evidence for both transcriptional and post transcriptional regulation of starch metabolism in Arabidopsis leaves. Plant Physiol 136: 2687-2699.

53. Haake V, Zrenner R, Sonnewald U, Stitt M (1998) A moderate decrease of plastid aldolase activity inhibits photosynthesis, alters the levels of sugars and starch, and inhibits growth of potato plants. Plant J 14: 147-157.

54. Henkes S, Sonnewald U, Badur R, Flachmann R, Stitt M (2001) A smal decrease of plastid Transketolase activity in antisense Tobacco transformants has dramatic effects on photosynthesis and phenylpropanoid metabolism. Plant Cell 13: 535-551.

55. Dinges JR, Colleoni C, James MG, Myers AM (2003) Mutational analysis of the pullulanase-type debranching enzyme of maize indicates multiple functions in starch metabolism. Plant Cell 15: 666-680.

\section{Appendix}

\begin{tabular}{|c|c|c|c|}
\hline Abbreviation & Name & Abbreviation & Name \\
\hline PGA & Phosphoglycerate & G6P & Glucose-6-phosphate \\
\hline BPGA & Bisphosphoglycerate & G1P & Glucose-1-phosphate \\
\hline GAP & Glyceraldehyde phosphate & $\mathrm{Pi}$ & Inorganic phosphate \\
\hline DHAP & Dihydroxy acetonephosphate & $\mathrm{PP}_{\mathrm{i}}$ & Pyrophosphate \\
\hline FBP & Fructose 1,6-bisphosphate & LBG & Large branched glucan (Branched $\alpha-1,4$-glucans) \\
\hline F6P & Fructose 6-bisphosphate & LLG & Long linear glucan (Linear a-1,4-glucans ) \\
\hline FUC & Fructose & MAL & Maltose \\
\hline E4P & Erythrose 4-phosphate & MALT & Maltotriose (Short glucans) \\
\hline SBP & Sedoheptulose 1,7-bisphosphate & UDPG & UDP-glucose \\
\hline S6P & Sucrose 6-phosphate & ADPG & ADP-glucose \\
\hline S7P & Sedoheptulose 7-phosphate & LG & Linear glucan \\
\hline X5P & Xylose 5-phosphate & PG & Phytoglycogen \\
\hline R5P & Ribose 5-phosphate & $\mathrm{AL}$ & Amylose \\
\hline Ru5P & Ribulose 5-phosphate & AP & Amylopectin \\
\hline GLC & Glucose & & \\
\hline
\end{tabular}

Table A1: Abbreviation of metabolites. 